\title{
The role of adsorbed hydroxide in hydrogen evolution reaction kinetics on modified platinum
}

\author{
Ian T. McCrum ${ }^{1,2}$ and Marc T. M. Koper (1) $1 \otimes$
}

\begin{abstract}
The bifunctional mechanism that involves adsorbed hydroxide in the alkaline hydrogen oxidation and evolution reactions, important in hydrogen fuel cells and water electrolysers, is hotly debated. Hydroxide binding has been suggested to impact activity, but the exact role of adsorbed hydroxide in the reaction mechanism is unknown. Here, by selectively decorating steps on a Pt single crystal with other metal atoms, we show that the rate of alkaline hydrogen evolution exhibits a volcano-type relationship with the hydroxide binding strength. We find that Pt decorated with $\mathrm{Ru}$ at the step edge is 65 times more active for the hydrogen evolution reaction (HER) than is the bare Pt step. Simulations of electrochemical water dissociation show that the activation energy correlates with the $\mathrm{OH}^{\star}$ adsorption strength, even when the adsorbed hydroxide is not a product, which leads to a simulated volcano curve that matches the experimental curve. This work not only illustrates the alkaline HER mechanism but also provides a goal for catalyst design in targeting an optimum hydroxide binding strength to yield the highest rate for the alkaline HER. A three-dimensional ( $\mathrm{H}$ and $\mathrm{OH}$ adsorbed species) HER activity volcano and the implications for hydrogen oxidation are discussed.
\end{abstract}

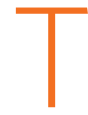
he hydrogen evolution and oxidation reactions (HER and HOR, respectively) are important electrocatalytic reactions and occur in hydrogen fuel cells and water electrolysers. In acidic electrolytes, a well-known correlation exists between the HER/HOR activity and the binding strength of hydrogen to the catalyst surface, which follows what is known as a 'volcano' trend ${ }^{1,2}$. Catalysts that bind hydrogen too strongly are limited by hydrogen desorption and those that bind hydrogen too weakly are limited by hydrogen adsorption; only catalysts with an intermediate binding strength give a high activity. The optimum adsorption energy is $0 \mathrm{eV}$ (with respect to the $\mathrm{H}_{2}$ molecule), as this generates a free-energy landscape without barriers or sinks ${ }^{2}$.

The kinetics of the HER/HOR in an alkaline electrolyte are more complex. It was found that the alkaline HER/HOR activities for $\operatorname{Ir}(111)$, polycrystalline Ir and a Pt-Ru alloy were much higher than those for $\mathrm{Pt}(111), \mathrm{Au}(111)$ and $\mathrm{Ru}(0001)$, and it was argued that this was due, in part, to a stronger hydroxide binding on the iridium and $\mathrm{Pt}-\mathrm{Ru}$ alloy surfaces ${ }^{3}$. This trend in activity, however, is also convoluted with the differing hydrogen binding strengths across these surfaces. Later work that examined the HER/HOR kinetics on $\mathrm{Pt}-\mathrm{Ru}$ alloys ${ }^{4}$ and $\mathrm{Pt}-\mathrm{Ru}$ core shell nanoparticles ${ }^{5}$ instead attributed the higher activity of these materials to a weakening, relative to that of pure platinum, of the hydrogen binding induced by ruthenium. This is expected to increase activity, as pure platinum sits on the too-strong binding side of the hydrogen volcano ${ }^{1,2}$.

Earlier work examined the kinetics of hydrogen evolution on a $\mathrm{Pt}(111)$ single-crystal electrode decorated with nanometre-size transition metal hydroxide clusters and/or islands ( $\mathrm{Ni}, \mathrm{Co}, \mathrm{Fe}$ and $\mathrm{Mn})$, and found that $\mathrm{Ni}(\mathrm{OH})_{2}$ and $\mathrm{Co}(\mathrm{OH})_{2}$ increased the rate of $\mathrm{HER}$, whereas $\mathrm{FeO}(\mathrm{OH})_{x}$ and $\mathrm{Mn}(\mathrm{OH})_{2}$ decreased the rate of $\mathrm{HER}^{6}$. By also examining the rates of $\mathrm{CO}$ stripping and the oxygen evolution reaction, which are known to involve adsorbed hydroxide, it was shown that the trend in HER activity follows the trend in hydroxide binding. Although this study removes the issue of a changing hydrogen binding strength (hydrogen is assumed to only adsorb on $\operatorname{Pt}(111)$ sites, and is unaffected by the presence of the transition metal hydroxide cluster), it has additional limitations. The hydroxide binding strength in the transition metal hydroxide clusters is not known and is difficult to calculate with density functional theory (DFT) ${ }^{6,7}$ due, in part, to difficulties in accurately modelling the electronic structure of transition metal ions, the size of each island being too large to explicitly model with DFT and the unknown nature of the active site $\left(\mathrm{OH}^{*}\right.$ adsorbed on $\mathrm{Pt}(111)$ near the cluster versus $\mathrm{OH}^{\star}$ adsorbed within the cluster, where the asterisk represents an adsorbed species). Additionally, the interfacial area between the edge of the island and the metal surface is unknown, and as the size and/or shape of the clusters changes ${ }^{6}$ between $\mathrm{Ni}, \mathrm{Co}$, $\mathrm{Fe}$ and $\mathrm{Mn}$, this would also affect the HER activity.

Furthering the complexity of alkaline HER/HOR is that the rates for these reactions are substantially lower in an alkaline electrolyte than in an acid electrolyte on many active catalysts, which include platinum $^{8-10}$. It was suggested that these $\mathrm{pH}$ effects are due, in part, to a change in electric field across the surface with $\mathrm{pH}$, which alters the energy of the reorganization of water near the surface ${ }^{11}$. This model is supported by showing that the increase in HER activity of $\mathrm{Pt}(111)$ on decoration with $\mathrm{Ni}(\mathrm{OH})_{2}$ nanoclusters is correlated with a decrease in the potential of maximum entropy (PME) and, consequently, in the potential of zero charge (PZC) of the electrode, which lowers the surface normal electric field under reaction conditions ${ }^{11}$.

There has since been substantial debate ${ }^{12,13}$ on the role of the electric field ${ }^{14}, \mathrm{PZC} / \mathrm{PME}^{15}$ and near-surface water ${ }^{16}$, with studies that either support ${ }^{17-20}$ or refute $e^{5,21,22}$ a bifunctional mechanism that involves adsorbed hydroxide, in the HER/HOR. However, the majority of this work was performed using complex polycrystalline or metal (alloy) nanoparticles, which made it difficult to connect changes in activity to an atomic-scale picture of the reaction mechanism, as the surface structure and composition, especially in the electrochemical environment, are unknown. Solving this debate requires the combination of detailed measurements on a

'Leiden Institute of Chemistry, Leiden University, Leiden, the Netherlands. ${ }^{2}$ Present address: Department of Chemical \& Biomolecular Engineering, Clarkson University, Potsdam, NY, USA. ${ }_{\text {e-mail: m.koper@lic.leidenuniv.nl }}$ 
a

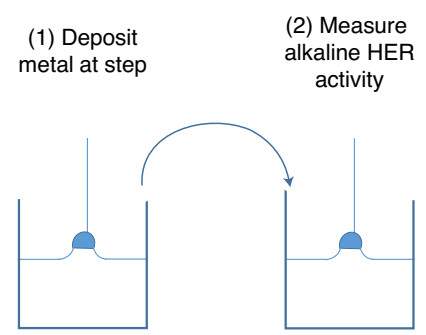

c

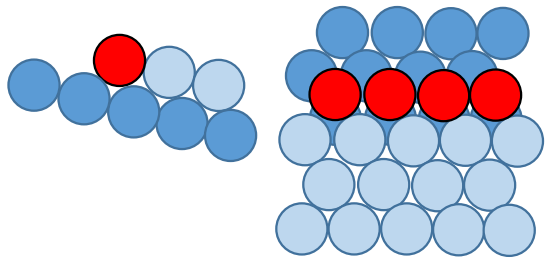

b

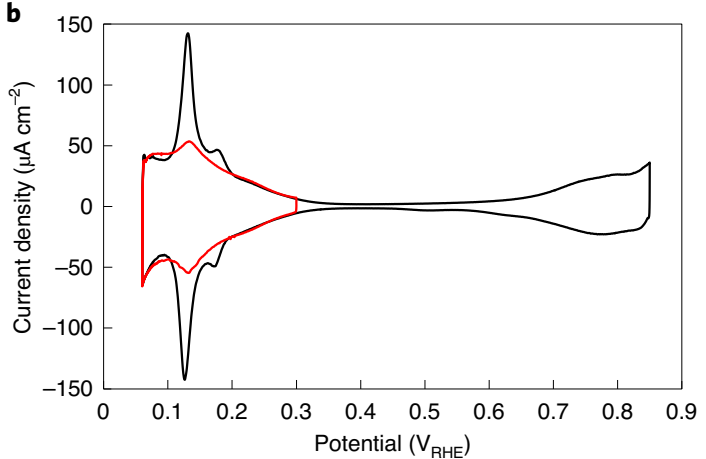

d

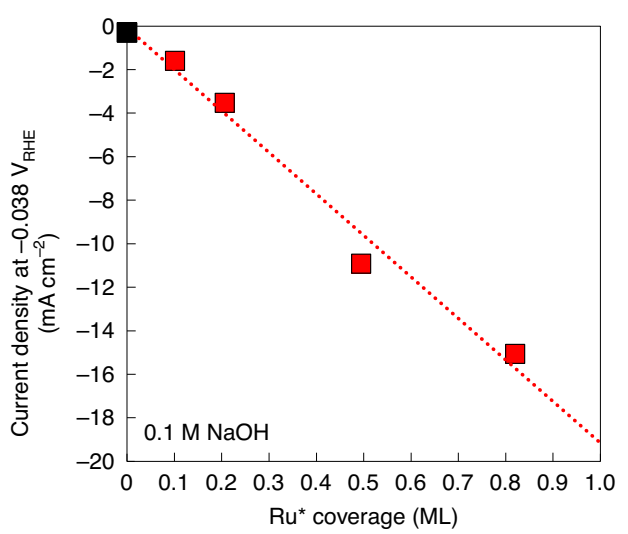

Fig. 1 | Step-decoration procedure, voltammetry and HER activity. a, Schematic of step decoration and HER activity measurement procedure. The steps of a $\mathrm{Pt}(553)$ single-crystal electrode were decorated electrochemically by cycling the electrode in an acid electrolyte $(0.1 \mathrm{M} \mathrm{HClO})$ that contained a low concentration $\left(10^{-5}\right.$ to $\left.10^{-6} \mathrm{M} \mathrm{M}^{x+}\right)$ of decorating metal ions. Both the step decoration and alkaline $(0.1 \mathrm{M} \mathrm{NaOH}) \mathrm{HER}$ activity measurements were performed using the hanging meniscus configuration. b, $\mathrm{CV}$ measured on $\mathrm{Pt}(553)$ in $0.1 \mathrm{M} \mathrm{HClO}_{4}$ (black) and after cycling in $0.1 \mathrm{M} \mathrm{HClO}_{4}+10^{-6} \mathrm{M} \mathrm{RuCl}_{3}$ (red), with the step decorated with Ru*. Voltammograms showing the same for other metals are given in Supplementary Fig. 1. c, Side (left) and top-down (right) views of the stepped Pt(553) showing Pt atoms forming an upper terrace (light blue), lower terrace (dark blue) and the step edge decorated with a metal adatom (red). d, Activity of hydrogen evolution (current density measured at $-0.038 \mathrm{~V}_{\mathrm{RHE}}$ (corrected for $i R$ )) as a function of Ru* coverage (red squares) and for bare Pt(553) (black square) showing a linear relationship, which supports that Ru* only decorates the step edge. The dashed red line is a linear regression of the data $\left(R^{2}=0.98\right)$.

well-defined surface with DFT modelling (of the reaction mechanisms) to which the measurements can be directly compared.

Here we aimed to establish a definite correlation between the HER activity and hydroxide binding strength, and understand why such a correlation might exist. To achieve this, we measured experimentally, and modelled using DFT, the HER kinetics in an alkaline electrolyte on a stepped $\mathrm{Pt}$ single-crystal electrode $(\mathrm{Pt}(553))$, in which the step was selectively decorated with $\mathrm{Mo}, \mathrm{Re}, \mathrm{Ru}, \mathrm{Rh}$ and Ag. Using this approach, we created a model catalyst surface with sites of variable $\mathrm{OH}^{\star}$ binding strength (at the step), constant hydrogen binding strength (on the terrace), a known and controllable interfacial area between these two regions, which is linearly proportional to the decorating atom coverage, and is sufficiently simple to model accurately using DFT. We also used DFT to investigate the role of an electric field and the PZC in HER kinetics.

Step decoration. Metal adatoms were deposited selectively at the Pt step edge by cycling the $\mathrm{Pt}(553)$ electrode from 0.06 to $0.35 \mathrm{~V}_{\mathrm{RHE}}$ (RHE, reversible hydrogen electrode) for $10-100$ cycles in $0.1 \mathrm{M}$ $\mathrm{HClO}_{4}$ that contained $10^{-5}$ to $10^{-6} \mathrm{M}$ metal cation. Similar procedures were used previously to deposit $\mathrm{Ru}$ and $\mathrm{Ag}$ onto stepped platinum $^{23,24}$. Figure 1 shows the step-decoration procedure, a model of a decorated $\mathrm{Pt}(553)$ surface and an example of how cyclic voltammograms (CVs) on $\mathrm{Pt}(553)$ change on step decoration with $\mathrm{Ru}^{*}$. CVs showing step decorations with $\mathrm{Mo}, \mathrm{Re}, \mathrm{Rh}$ and $\mathrm{Ag}$ are given in
Supplementary Fig. 1. The CV in $0.1 \mathrm{M} \mathrm{HClO}_{4}$ in Fig. 1 b shows three features: a broad peak due to hydrogen adsorption on (111)-like terrace sites (0.06-0.3 $\mathrm{V}_{\mathrm{RHE}}$ ), a sharp peak due to an exchange between hydrogen and co-adsorbed hydroxide and water at the (110) step edge $\left(0.125 \mathrm{~V}_{\mathrm{RHE}}\right)$ (ref. $\left.{ }^{25}\right)$ and a peak at high potentials due to the adsorption of hydroxide on the (111)-like terrace $\left(0.6-0.85 \mathrm{~V}_{\mathrm{RHE}}\right)$. The voltammogram measured after the deposition of $\mathrm{Ru}$ shows that the $\mathrm{Ru}^{*}$ blocks the adsorption of the hydrogen and hydroxide at the Pt step edge, which eliminates the sharp peak at $0.125 \mathrm{~V}_{\mathrm{RHE}}$. The peak due to hydrogen adsorption on the terrace is unaffected, which indicates that Ru is deposited only at the step edge, and that its presence at the step edge does not affect the binding strength of hydrogen on the terrace. Similar results are seen with the other metal adatoms. DFT calculations support that the presence of Re, $\mathrm{Ru}$ and $\mathrm{Rh}$ at the step have only a small or negligible effect on the binding strength of hydrogen to the terrace (Supplementary Fig. 2). $\mathrm{Ru}^{*}$ at the step edge additionally has little effect on the adsorption of hydroxide at the terrace ${ }^{26}$. We found with both experiment and DFT that the presence of $\mathrm{Ag}^{\star}$ at the step edge does affect the binding of hydrogen at the terrace, although this did not impact the interpretation of our results. Figure 1d gives the rate of the HER on the $\mathrm{Ru}$-decorated $\mathrm{Pt}(553)$ as a function of $\mathrm{Ru}^{\star}$ coverage. Importantly, the relationship is linear, which supports that the interfacial area between hydrogen adsorbed on the terrace and hydroxide adsorbed on the $\mathrm{Ru}^{\star}$ is linearly related to the coverage of $\mathrm{Ru}^{\star}$ and that $\mathrm{Ru}^{*}$ 


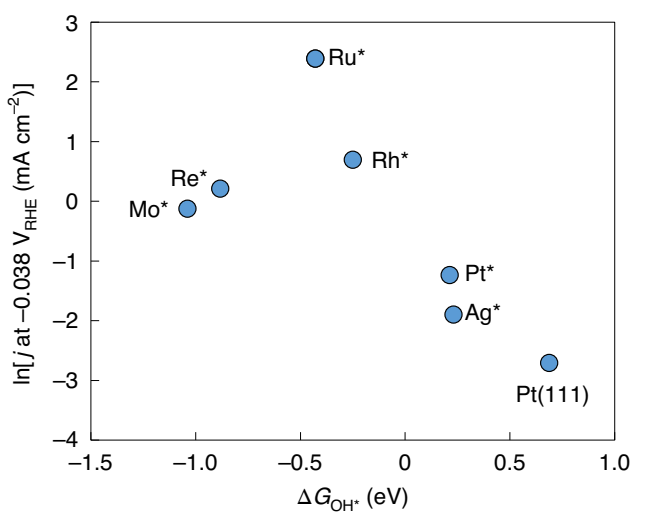

Fig. 2 | Experimentally measured HER activity on step-decorated Pt(553). Natural logarithm of the experimentally measured rate of hydrogen evolution $j$ (measured at $-0.038 \mathrm{~V}_{\mathrm{RHE}}$, corrected for $\mathrm{R} R$ ) on $\mathrm{Pt}(553)$ and on $\mathrm{Pt}(553)$ with a high coverage of $\mathrm{Mo}^{\star}, \mathrm{Re}^{\star}, \mathrm{Ru}^{\star}, \mathrm{Rh}^{\star}$ and $\mathrm{Ag}^{\star}$ adsorbed at the step edge, plotted against the DFT-calculated hydroxide adsorption free energy at $\mathrm{OV}_{\mathrm{RHE}}$. $\mathrm{Pt}(111)$ is also shown for comparison.

islands or clusters are not formed, as these would contribute to a non-linear dependence of the activity on $\mathrm{Ru}^{*}$ coverage. The activity of the $\mathrm{Ru}^{\star}$-decorated step edge, extrapolated to a complete 1 monolayer (ML, defined per step Pt atom) coverage, is 65 times that of the bare $\operatorname{Pt}(553)$ surface.

HER activity. Having established that these metals can be selectively deposited at the step edge without affecting the binding strength of hydrogen on the terrace, their effect on HER kinetics and hydroxide adsorption at the step edge was evaluated. Figure 2 illustrates that the experimentally measured rate of the HER in an alkaline electrolyte follows a volcano type relationship with the DFT-calculated binding strength of hydroxide on the adatom at the step. The HER activity is measured at similar adatom coverages, given in Supplementary Table 1 (calculated from voltammograms measured in the alkaline electrolyte and shown in Supplementary Fig. 3). Polarization curves (from which the HER activity was measured) are given in Supplementary Fig. 4, and replicate HER activity measurements are given in Supplementary Fig. 5. Hydroxide-adsorption free energies were calculated at a full $1 \mathrm{ML}$ coverage of the step-decorating atom using DFT.

Water dissociation kinetics. Experimentally measured Tafel slopes for HER on platinum suggest the first step in the mechanism is rate determining and involves electron transfer $\left(\sim 120 \mathrm{mV} \mathrm{dec}^{-1}\right)$ (refs $8,21,27$ ), which rules out a mechanism that involves fully discharged adsorbed hydroxide as a reaction intermediate ${ }^{21}$. To better understand the cause for the correlation shown in Fig. 2, we examined the barrier for water dissociation (the first electron transfer step, also called the alkaline Volmer step) at the step edge using DFT for the undecorated step edge and the step edges decorated with $\mathrm{Re}, \mathrm{Ru}, \mathrm{Rh}$ and Ag. Figure 3a shows how the activation energy for water dissociation is linearly correlated with the binding strength of the adsorbed hydroxide at the step edge (and on Pt(111), included for comparison). Activation energies were calculated for a product state that involved adsorbed hydroxide (non-electrochemical) or solution-phase hydroxide (electrochemical, the alkaline Volmer step). The activation energies for dissociation to solution-phase hydroxide were calculated by taking the barrier for the reaction to the adsorbed hydroxide as the potential-dependent barrier for the reaction to solution-phase hydroxide at the potential at which the solution-phase hydroxide and the adsorbed hydroxide are in equilibrium ${ }^{28}$. These barriers were taken to be the same for metals that have an adsorption potential of hydroxide below $0 \mathrm{~V}_{\mathrm{RHE}}$ ( $\mathrm{Re}, \mathrm{Ru}$ and $\mathrm{Rh}$ ), as hydroxide adsorption is favourable on these metals at the equilibrium potential for hydrogen evolution $\left(0 \mathrm{~V}_{\mathrm{RHE}}\right)$. The barriers for water dissociation on the undecorated step edge, on $\mathrm{Ag}^{\star}$ on $\mathrm{Pt}(553)$ and on $\mathrm{Pt}(111)$ are lower for water dissociation that proceeds to give the solution-phase hydroxide; the unfavourable adsorption of hydroxide at $0 \mathrm{~V}_{\mathrm{RHE}}$ contributes to an increased barrier for the reaction that proceeds to the adsorbed hydroxide. This also results in a small slope (weaker correlation) between the hydroxide adsorption strength and the barrier for (electrochemical) water dissociation.

The linear correlation between the activation energy for water dissociation and the hydroxide adsorption energy can be considered as a Brønsted-Evans-Polyani relationship. The cause for this is illustrated in Fig. 3b. As the reaction path was essentially identical for all the metal adatoms adsorbed at the step edge and followed a one dimensional (1D) reaction coordinate, we can plot the energy along the reaction path as a function of this reaction coordinate. Taking this coordinate to be the $\mathrm{O}-\mathrm{H}$ bond length (for the bond that is dissociated), this energy is plotted in Fig. $3 \mathrm{~b}$ and an image of a representative transition state (TS) is given in Fig. 3c. Figure $3 b$ illustrates that, as the adsorbed hydroxide is stabilized at the step edge (a more-negative adsorption energy), the activation energy for dissociation decreases as the intersection in energy between the reactant and product states moves to lower energies. This intersection point also moves earlier along the reaction coordinate with a stronger $\mathrm{OH}^{\star}$ binding, which yields a shorter $\mathrm{O}-\mathrm{H}$ bond length at the TS (earlier TS) with a stronger $\mathrm{OH}^{*}$ binding. We found the metal-oxygen bond lengths at both the initial state (IS) $\left(\mathrm{H}_{2} \mathrm{O}^{*}\right)$ and final state (FS) $\left(\mathrm{OH}^{*}\right)$ correlate with both the $\mathrm{OH}^{*}$ binding strength and the activation energy for dissociation (Supplementary Fig. 6).

Alkaline HER reaction mechanism. The DFT-calculated kinetics shown in Fig. 3a (and the experimentally measured volcano trend in Fig. 2) allow us to determine the mechanism of the HER in an alkaline electrolyte. Given that both the experimentally measured rate and the barrier for water dissociation are correlated with the hydroxide adsorption strength, the rate-determining step (RDS) on the 'too-weak' binding side of the volcano must be water dissociation into adsorbed hydrogen and solution-phase hydroxide. This is supported by experimentally measured Tafel slopes (Supplementary Fig. 7, $120 \mathrm{mV} \mathrm{dec}^{-1}$ ). A RDS that involves hydrogen recombination (to $\mathrm{H}_{2}$ gas, a 'Tafel' step) would not involve an explicit electron transfer or a dependence on hydroxide binding strength. On the 'too strong' binding side of the volcano, the RDS must be hydroxide desorption. This is supported by the DFT-calculated barriers, which suggest the barrier for water dissociation on $\mathrm{Re}^{\star}$ is small and, within reasonable error, effectively zero. Extrapolating the trend in Fig. 3a, we expect water dissociation on catalysts that bind $\mathrm{OH}^{\star}$ more strongly than $\mathrm{Re}^{\star}$ to be non-activated. On the too-weak binding side of the $\mathrm{OH}^{\star}$ volcano, the reaction mechanism follows equations (1) and (2), with the Volmer step (equation (1)) being the RDS:

$$
\begin{gathered}
\mathrm{H}_{2} \mathrm{O}+^{*}+e^{-} \rightarrow \mathrm{H}^{*}+\mathrm{OH}^{-} \text {RDS } \\
\mathrm{H}^{*} \rightarrow^{\star}+\frac{1}{2} \mathrm{H}_{2}
\end{gathered}
$$

This mechanism yields the rate equations (3) and (4):

$$
\begin{gathered}
\text { Rate }=k_{\mathrm{W}}\left(1-\theta_{\mathrm{H}^{*}}\right) \\
\text { Rate }=\frac{A \exp \left(\frac{-\Delta G_{\mathrm{W}}^{\mathrm{TS}}}{R T}\right)}{1+\exp \left(\frac{-\Delta G_{\mathrm{H}^{*}}}{R T}\right)}
\end{gathered}
$$


a

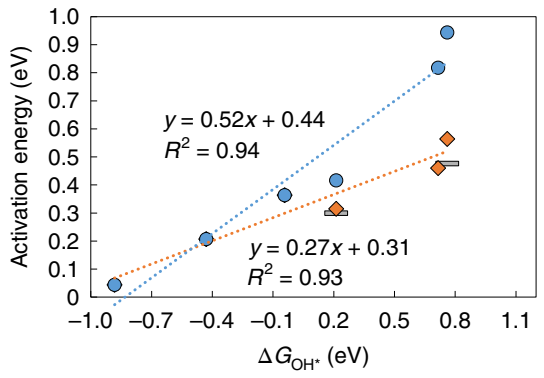

b

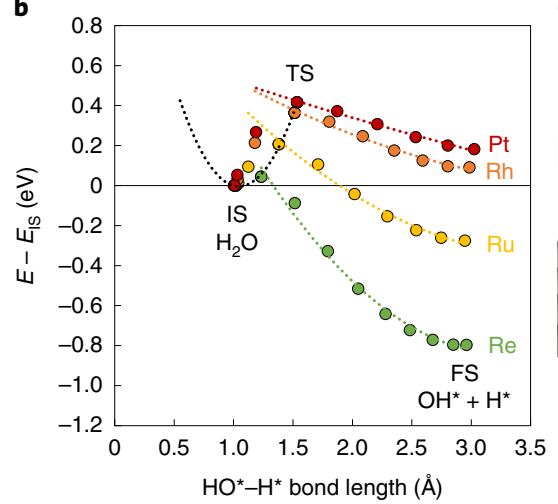

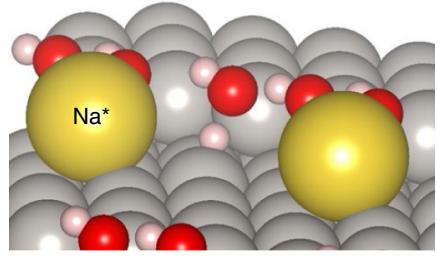

Fig. 3 | DFT-simulated activation energy of water dissociation (the alkaline Volmer reaction). a, DFT-calculated activation energies for water dissociation on $\mathrm{Pt}$ (111), $\mathrm{Pt}$ (553) and $\mathrm{Pt}$ (553) decorated with $1 \mathrm{ML}$ of $\mathrm{Re}^{\star}, \mathrm{Ru}^{\star}, \mathrm{Rh}^{\star}$ or $\mathrm{Ag}^{\star}$ at the step, calculated with $\mathrm{OH}^{\star}$ as a product (blue circles) and with $\mathrm{OH}^{-}$as a product (orange diamonds), extrapolated to $O V_{R_{H E}}$ plotted against the adsorption energy of hydroxide (calculated in the presence of adsorbed hydrogen on the terrace in the product state). The dashed lines are linear regressions of the simulated activation energy for $\mathrm{OH}^{\star}$ as a product (blue) and $\mathrm{OH}^{-}$as a product (orange). Experimentally measured activation energies for hydrogen evolution (grey rectangles) on Pt(111) (ref. ${ }^{53}$ ) and $\mathrm{Pt}(\mathrm{polycrystalline)}$ (shown at the hydroxide adsorption energy of $\mathrm{Pt}(553))^{8}$ are given for comparison. $\mathbf{b}$, Energy along the reaction coordinate relative to each IS $\left(\mathrm{H}_{2} \mathrm{O}\right.$ near the surface) energy for $\mathrm{Pt}$ (553) and $\mathrm{Pt}(553)$ with $\mathrm{Re}^{\star}, \mathrm{Ru}^{\star}$ and $\mathrm{Rh}^{\star}$. The dashed lines represent a quadratic regression, intended only as a guide for the eye. c, Image of the structure of the TS for water dissociation at the Pt(553) step, which shows Pt (grey), O (red), H (white) and Na (yellow). The effect of a near-surface cation $\left(\mathrm{Na}^{\star}\right)$ is included, as our previous work showed the cation to strongly influence water and hydroxide adsorption at the step ${ }^{50}$. The IS, TS and FS structures for water dissociation on each surface are given in Supplementary Figs. 15-20.

where $k_{\mathrm{w}}$ is the rate constant for the Volmer step (equation (1)), $\theta_{\mathrm{H}^{*}}$ the coverage of hydrogen adsorbed on the terrace, $\Delta G_{\mathrm{W}}^{\mathrm{TS}}$ the activation energy of the Volmer step, $\Delta G_{\mathrm{H}^{*}}$ the adsorption energy of hydrogen on the terrace and $A$ is the pre-exponential factor (for equation (1)). On the too-strong binding side of the $\mathrm{OH}^{\star}$ volcano, the reaction follows equations (5)-(7) to yield rate equations (8) and (9):

$$
\begin{gathered}
\mathrm{H}_{2} \mathrm{O}+2^{*} \rightarrow \mathrm{H}^{*}+\mathrm{OH}^{*} \\
\mathrm{H}^{*} \rightarrow^{*}+\frac{1}{2} \mathrm{H}_{2} \\
\mathrm{OH}^{*}+e^{-} \rightarrow \mathrm{OH}^{-}+{ }^{*} \mathrm{RDS} \\
\text { Rate }=k_{\text {des }}\left(\theta_{\mathrm{OH}^{*}}\right) \\
\text { Rate }=A \exp \left(\frac{\Delta G_{\mathrm{OH}^{*}}}{R T}\right)
\end{gathered}
$$

where $k_{\text {des }}$ is the rate constant for $\mathrm{OH}^{\star}$ desorption and $\theta_{\mathrm{OH}^{*}}$ the coverage of hydroxide adsorbed at the step and $\Delta G_{\mathrm{OH}^{*}}$ the free energy of adsorption of hydroxide, where we have assumed that the activation barrier for hydroxide desorption is equal to or larger than the desorption free energy of hydroxide, which is $-\Delta G_{\mathrm{OH}^{*}}$. With this assumption, in conjunction with the DFT-calculated correlation between the activation energy for the alkaline Volmer step and the hydroxide binding strength (Fig. 3a) (the $0 \mathrm{~K}$ enthalpic barrier, $\Delta E_{\mathrm{W}}^{\mathrm{TS}}$, which we take to be an approximation of $\Delta G_{\mathrm{W}}^{\mathrm{TS}}$ ), we can simulate the rate of hydrogen evolution for any arbitrary hydroxide adsorption strength. We examine first only a constant hydrogen binding energy, calculated using DFT for a low hydrogen coverage on the terrace of $\mathrm{Pt}(553)$.

Figure 4 shows the simulated HER activity and the experimentally measured activity, both plotted as a function of the hydroxide adsorption strength. There is good agreement between the theory and experiment. In addition to the volcano-type trend in activity, there is also a change in the experimentally measured Tafel slopes (Supplementary Fig. 7) between metals on the too-weak binding side of the volcano (105-157 $\mathrm{mV} \mathrm{dec}^{-1}, \mathrm{Rh}^{\star}, \mathrm{Pt}, \mathrm{Ag}^{\star}$ and $\mathrm{Pt}(111)$ ) to those on the too-strong binding side of the volcano $\left(41-75 \mathrm{mV} \mathrm{dec}^{-1}\right.$, $\mathrm{Mo}^{*}, \mathrm{Re}^{*}$ and $\left.\mathrm{Ru}^{*}\right)$, consistent with a change in the RDS.

On the too-strong binding side of the volcano, the experimentally measured activities for $\mathrm{Re}^{\star}$ and $\mathrm{Mo}^{*}$ appear to be far from the simulated volcano. However, given that for these adatoms water dissociation is not rate limiting, these step edges could be covered with (a high coverage of) adsorbed hydroxide or even oxygen. If we calculate the reaction energy to partially oxidize the $\mathrm{Re}^{\star}$ and $\mathrm{Mo}^{\star}$ step edge, the experimentally measured points move much closer to the simulated volcano. Additionally, the prefactor for $\mathrm{OH}^{*}$ desorption, which we assumed to be equal to that for water dissociation, could be larger and shift the 'too-strong' binding side of the volcano to higher rates, or our model to describe $\mathrm{OH}^{*}$ adsorption thermodynamics may be oversimplified for strong-binding adatoms.

We also performed a preliminary investigation into the role of an electric field and the electrode PZC in the HER kinetics and reaction mechanism. Although we expect water reorganization energetics and the electrode PZC to be important, and cannot rule out that the electrode PZC may be correlated with the hydroxide binding strength, we conclude that for the system studied here, the adsorption strength of the hydroxide is a more useful descriptor. Additionally, it is difficult to explain the evidence for the change in reaction mechanism (between the too-strong and too-weak binding sides of the $\mathrm{OH}^{\star}$ volcano) following the water reorganization model. See Supplementary Note 1 (and Supplementary Figs. 8-10) for a more detailed discussion. We note that the adsorption strength of hydroxide is easier to simulate and measure experimentally (than the electrode PZC) and chemical intuition can be more readily used for catalyst design (more-oxophilic elements may promote $\mathrm{OH}^{*}$ adsorption, for example).

For catalysts with a stronger or weaker hydrogen binding strength (than that considered in Fig. 4), the activity and, importantly, the binding strength of hydroxide that yields the highest activity would also change. For a hydrogen free energy of adsorption of $0 \mathrm{eV}$, our model in Fig. 4 yields an optimum hydroxide free 

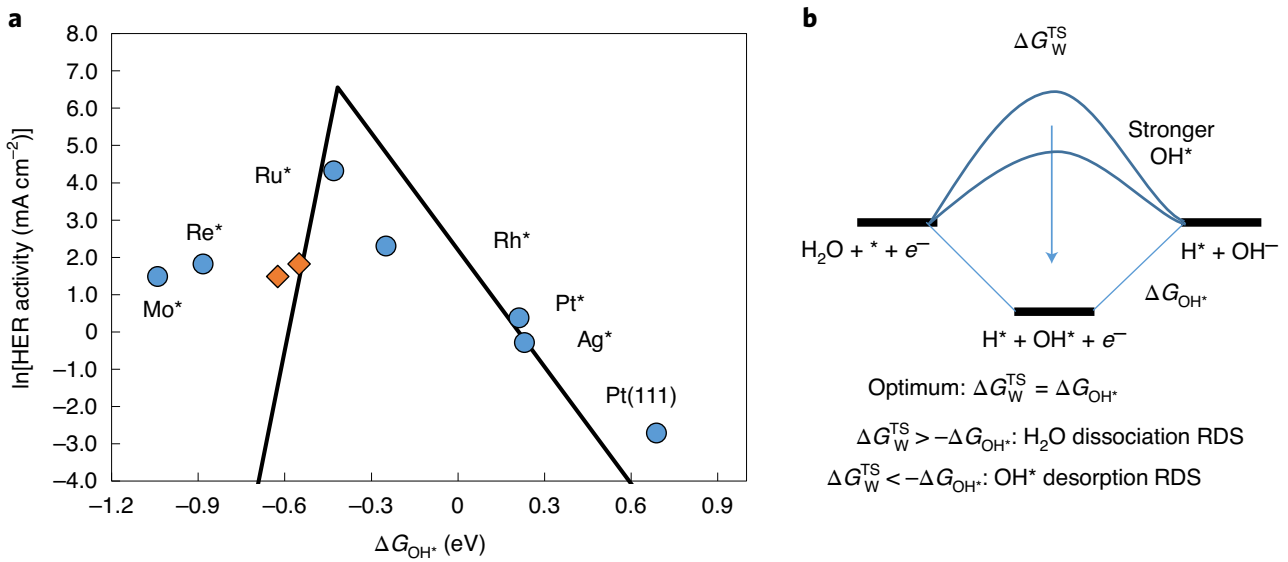

Fig. 4 | Simulated and experimentally measured HER activity and HER reaction mechanism. a, Plot of the simulated (black line) and experimentally measured (blue circles) rates of hydrogen evolution on Pt(553), Pt(553) with $\mathrm{Mo}^{\star}, \mathrm{Re}^{\star}, \mathrm{Ru}^{\star}, \mathrm{Rh}^{\star}$ and $\mathrm{Ag}^{\star}$ adsorbed at the step and Pt(111) versus the DFT calculated hydroxide adsorption energy at $O \mathrm{~V}_{\mathrm{RHE}}$. The orange diamonds correspond to the experimentally measured rate on the $\mathrm{Mo}^{\star}$ and $\mathrm{Re}^{\star}$ decorated steps plotted against the DFT-calculated potential to oxidize the step $\left(1 / 3 \mathrm{MLOH}^{\star}+2 / 3 \mathrm{MLO}^{\star}\right.$ on $\mathrm{Re}^{\star}$ and $1 \mathrm{MLO}^{\star}$ on $\left.\mathrm{Mo}^{\star}\right)$. The rate of HER is simulated by using the DFT-calculated water dissociation barrier, $\Delta G_{W}^{\text {TS }}$ (which we approximate with the $0 \mathrm{~K}$ enthalpic barrier, $\Delta E_{\mathrm{W}}^{\mathrm{TS}}$, from Fig. 3a), the DFT-calculated hydrogen adsorption energy on the $\mathrm{Pt}(553)$ terrace (constant) and the hydroxide adsorption energy $\Delta G_{\mathrm{OH}^{*}}$. The HER rate is measured at $-0.038 \mathrm{~V}_{\mathrm{RHE}}$ and simulated at $\mathrm{OV}_{\mathrm{RHE}}$. b. Reaction energy diagram to illustrate the HER reaction mechanism. On the too-weak binding side of the $\mathrm{OH}^{*}$ volcano, the rate is limited by water dissociation (the Volmer step). As the strength of the $\mathrm{OH}^{\star}$ binding increases (downward pointing arrow), $\mathrm{OH}^{\star}$ is adsorbed and $\mathrm{OH}^{\star}$ desorption becomes the RDS.

energy of adsorption of $-0.26 \mathrm{eV}$. Discussion of these points is given in subsequent sections. To gain additional insight, we can compare this DFT-simulated volcano to both the traditional hydrogen binding volcano for HER/HOR in acidic media and to the kinetics of water dissociation and hydronium dissociation in solution.

It was recently found that the activation barrier to adsorb hydrogen from water (alkaline Volmer step) on $\mathrm{Pt}(111)$ is much higher than that to adsorb hydrogen from hydronium ${ }^{29}$ (acid Volmer step), which matches the trend seen for water and hydronium dissociation in solution (Supplementary Fig. 11) ${ }^{30}$. This high barrier to adsorb hydrogen from water is, in part, why the hydroxide-binding volcano in Fig. 4 is centered around the relatively strong $\mathrm{OH}^{\star}$ binding (see discussion in Supplementary Note 2) and the activity of many catalysts for HER is much lower (and the activation energy much higher ${ }^{8,31}$ ) in an alkaline electrolyte than in an acid electrolyte. Figures 2-4 show that by promoting hydroxide adsorption (relative to $\mathrm{Pt}$ ), the activity in an alkaline electrolyte can be increased.

Effect of site blocking and/or co-adsorbates at the step. On the too-strong binding side of the $\mathrm{OH}^{\star}$ volcano $\left(\mathrm{Ru}^{*}, \mathrm{Re}^{\star}\right.$ and $\left.\mathrm{Mo}^{*}\right)$, the rate is limited by the desorption of strongly bound hydroxide; it is the dominant adsorbate ( $\mathrm{and} / \mathrm{or} \mathrm{O}^{*}$, as considered for Mo and $\mathrm{Re}$ ). On the too-weak binding side of the $\mathrm{OH}^{*}$ volcano, however, we had considered the sites available for $\mathrm{H}^{\star}$ adsorption on the terrace, but we had not considered the sites available at the step where water dissociation occurs. We calculated the barrier for water dissociation with DFT (Fig. 3) on an empty step edge, with only the effect of co-adsorbed water considered. In reality, the steps could be covered with a high coverage of hydrogen or hydroxide under the reaction conditions at $0 \mathrm{~V}$ versus RHE, as most adsorbates bind strongly to the adatoms at the step (except for on $\mathrm{Ag}^{\star}$ ) and we might expect the adsorption on the step, because it is so strong, to be fast (compared with the adsorption of $\mathrm{H}^{\star}$ on the terrace). Supplementary Fig. 12 shows the adsorption energy of hydrogen and hydroxide at the step for the bare and decorated Pt(553). Supplementary Fig. 13a,b shows the calculated coverage of vacant step sites (assuming that the adsorption of $\mathrm{H}^{\star} / \mathrm{OH}^{*}$ at the step is fast and equilibrated) and the rate of HER given this site-blocking effect, respectively. The trend in HER activity (with $\mathrm{OH}^{*}$ binding strength across the adatoms) is identical to that shown in Fig. 4. This is a consequence of the much larger difference in $\mathrm{OH}^{*}$ binding strength across these metals than that of the $\mathrm{H}^{\star}$ binding strength, which makes the increase in the water-dissociation rate constant larger than the decrease in vacant sites with stronger binding on the too-weak binding side of the $\mathrm{OH}^{*}$ volcano. In addition to this simple model, there is still a need for more-comprehensive steady-state models and DFT simulations $s^{7,32}$ to map out more completely the exact coverage and binding sites of hydrogen and hydroxide on both the decorated step and Pt terrace and their effect on water dissociation under the reaction conditions.

Implications for catalyst design. A complete alkaline hydrogen evolution 3D volcano plot is shown in Fig. $5^{33}$. This gives the rate of hydrogen evolution as a function of hydrogen binding strength and hydroxide binding strength. The activation energy for water dissociation is calculated using data from a previous study ${ }^{34}$. The activation energies for hydrogen recombination (Tafel step) and $\mathrm{OH}^{\star}$ desorption are assumed to be equal to the energy of desorption of hydrogen and of hydroxide, respectively, and the kinetic prefactors are assumed to be the same for all the reactions. Given the limited dataset used to calculate the water dissociation barrier, and the many assumptions necessary to make this plot, we suggest it only as a guide. Figure 5 qualitatively captures trends in alkaline HER kinetics and gives guidelines for catalyst design. To improve the performance relative to $\mathrm{Pt}$, for example, we need to weaken the hydrogen adsorption strength (by $\sim 0.2 \mathrm{eV}$ ) and substantially promote the hydroxide adsorption strength (by $\sim 0.9 \mathrm{eV}$ relative to $\mathrm{Pt}(111)$ or by $\sim 0.3 \mathrm{eV}$ relative to $\mathrm{Pt}(553)$ ).

The reaction mechanism shown in Fig. 4 and resultant 3D volcano in Fig. 5 explain the bifunctionality of hydrogen evolution. On the too-strong binding side of the volcano, the reaction is bifunctional as it involves both adsorbed hydrogen and adsorbed hydroxide. On the too-weak binding side of the volcano, hydrogen evolution is only apparently bifunctional; adsorbed hydroxide is not necessarily a reaction intermediate, but the activation energy of the RDS is correlated with the $\mathrm{OH}^{*}$ binding strength. The 3D volcano 


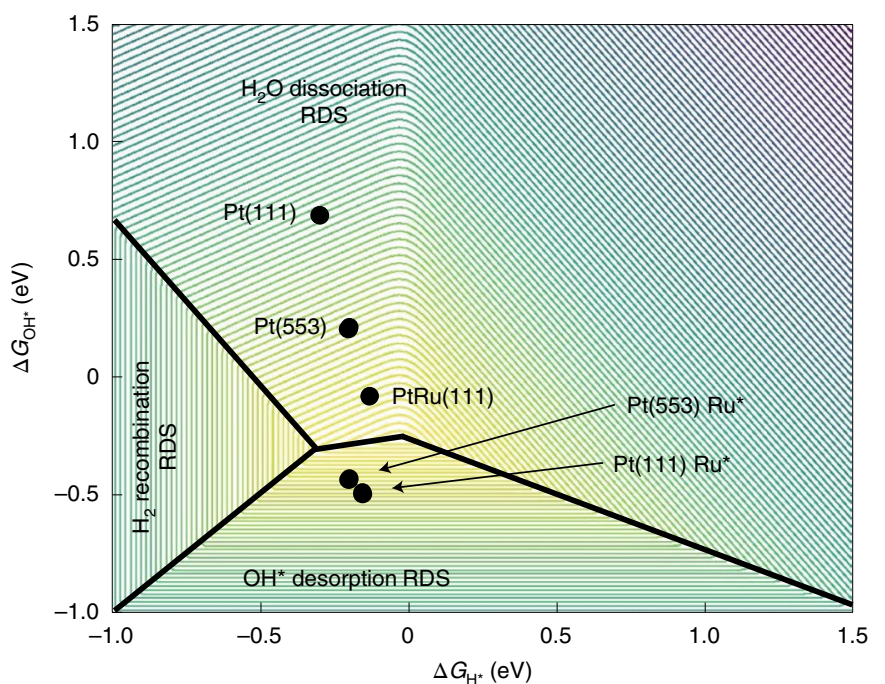

Fig. 5 | 3D HER activity volcano for catalyst design. Logarithm of the rate of hydrogen evolution (contours) as a function of hydrogen binding energy and hydroxide binding energy. The water dissociation rate was derived from the binding energies and kinetics on (211) surfaces ${ }^{34}$ calculated previously and modified to reference solution-phase hydroxide as the product of water dissociation and then extrapolated to $0 \mathrm{~V}_{\text {RHE }}$ and to reproduce the barriers calculated here for water dissociation on Pt(111) and Pt(553) (which include the effects of solvation and alkali cation). Black circles correspond to DFT-calculated hydrogen and hydroxide adsorption energies on Pt(111), Pt(553), Ru* adsorbed at the step of Pt(553), a PtRu(111) alloy and $\mathrm{Ru}^{\star}$ clusters on $\mathrm{Pt}(111)$. Catalysts that bind hydrogen at an intermediate strength at $0 V_{\mathrm{RHE}}($ near $\mathrm{OeV}$ ) and hydroxide strongly (near $-0.3 \mathrm{eV}$ ) yield the highest rates of hydrogen evolution (yellow). Catalysts with weak hydrogen binding and weak hydroxide binding (top right) yield the lowest rates (purple).

plot shown in Fig. 5 also helps to clarify the debate over the mechanism for the improved performance of $\mathrm{Pt}-\mathrm{Ru}$ alloy materials ${ }^{3-5}$ and $\mathrm{Ru}$ clusters and/or islands on Pt(111) (ref. ${ }^{35}$ ) - both weakened hydrogen binding and stronger hydroxide binding contribute.

Implications for hydrogen oxidation. Although we have not studied the HOR (the reverse of hydrogen evolution), we can consider what our proposed reaction model for hydrogen evolution might tell us about hydrogen oxidation. We assume that the reaction path is the same as that for hydrogen evolution and limit the discussion to catalysts that lie on the too-strong binding side of the hydrogen binding volcano (where hydrogen dissociation and/or recombination is not rate limiting, such as for stepped $\mathrm{Pt}$ ). In short, we find that, although we expect the rate of the HOR to be equal to that of the HER on the too-weak binding side of the $\mathrm{OH}^{*}$ volcano (a change in rate constant cancels with a change in the rate equation (Supplementary Fig. 14)), we also expect a change in the RDS between the HER and the HOR on the too-strong binding side of the $\mathrm{OH}^{\star}$ volcano, which means the rate of the HOR can be different from that of the HER (Supplementary Fig. 14). For a catalyst that has a favourable adsorption energy of hydrogen $(\Delta G<0)$, such as for $\mathrm{H}^{\star}$ on the terrace of stepped $\mathrm{Pt}$, and that sits on the too-strong side of the $\mathrm{OH}^{\star}$ volcano, this results in a lower rate of the HOR than that of the HER for the same $\mathrm{OH}^{\star}$ binding strength. A consequence of this is that there exists catalysts that have a stronger $\mathrm{OH}^{\star}$ binding strength than, for example, $\mathrm{Pt}$, and show a higher rate of hydrogen evolution, but a lower rate of hydrogen oxidation relative to Pt (and not the other way around) (catalyst (b) in Supplementary Fig. 14) in an alkaline electrolyte. This selective promotion of the
HER and not the HOR (as well as the promotion of both HER and $\mathrm{HOR}$ via a stronger $\mathrm{OH}^{\star}$ binding (catalyst (a) in Supplementary Fig. 14)) has been observed experimentally $y^{3,18,22,36}$, and the reaction model we present here in Supplementary Fig. 14 may explain this phenomenon. An additional consequence is that there are different optimum $\mathrm{OH}^{\star}$ binding strengths that yield the highest rate for the HER and for the HOR; to quantify this optimum for the HOR requires further study.

\section{Conclusions}

From this work, we not only determined the importance of hydroxide binding strength and the mechanism of alkaline HER but also provided insight for catalyst design. We quantified an optimum hydroxide binding strength and showed that the hydrogen binding strength and hydroxide binding strength must both be optimized to improve catalyst activity. This provides a new dimension, with a specific goal, to design more active and low-cost (non-noble metal) HER catalysts.

\section{Methods}

Electrochemical cell and electrolyte preparation. Measurements in the alkaline electrolyte $(0.1 \mathrm{M} \mathrm{NaOH})$ were performed in a fluorinated ethylene propylene electrochemical cell; those in an acid electrolyte $\left(0.1 \mathrm{M} \mathrm{HClO}_{4}\right)$ were performed in a glass cell. Before each experiment, all the glassware and plasticware was cleaned by first storing overnight in a dilute, acidic solution of potassium permanganate. The glassware or plasticware was then rinsed with ultrapure water, soaked in a dilute acidic solution of hydrogen peroxide and then boiled in and rinsed with ultrapure water five times. The electrolyte was prepared with ultrapure water and concentrated perchloric acid (traceSELECT, Fluka) or concentrated sodium hydroxide (Suprapur, Merck). Bead-type single-crystal electrodes were used as the working electrode using the hanging meniscus technique, with a platinum wire counter electrode and reversible hydrogen reference electrode.

Single-crystal electrode preparation. Bead-type $\mathrm{Pt}(111)$ and $\mathrm{Pt}(553)$ electrodes were prepared following the Clavilier method $^{37}$ before every experiment; the electrode was flame annealed for $1 \mathrm{~min}$, allowed to cool in an $\mathrm{Ar} / \mathrm{H}_{2}$ (3:1) atmosphere and then dipped into ultrapure water saturated with the same gas mixture. Transfer through air to the electrochemical cell occurred while the surface of the electrode was protected with a droplet of water.

Metal adatom deposition. Metal adatoms were deposited selectively at the step edge by transferring the electrode to a perchloric acid electrolyte $(0.1 \mathrm{M})$ that contained $10^{-4}$ to $10^{-7} \mathrm{M} \mathrm{Mo}^{7+}, \mathrm{Re}^{7+}, \mathrm{Ru}^{3+}, \mathrm{Rh}^{3+}$ or $\mathrm{Ag}^{+}$ions and cycling between 0.05 and $0.35 \mathrm{~V}_{\mathrm{RHE}}$ for 10-200 cycles to deposit the metal atom at the step edge. The concentration and number of cycles were varied to control the amount of metal atom deposited at the step. Mo, Re, $\mathrm{Ru}, \mathrm{Rh}$ and $\mathrm{Ag}$ solutions were made from ultrapure water and sodium molybdate (Sigma Aldrich), ammonium perrhenate (Johnson Matthey), ruthenium chloride (Aldrich Chemie), rhodium chloride (Johnson Matthey) or silver perchlorate (Sigma Aldrich). Metal adatoms on $\mathrm{Pt}(553)$ were removed by repeatedly dipping the electrode in concentrated nitric acid and annealing in air. The adsorptions of $\mathrm{Ni}, \mathrm{Co}, \mathrm{Fe}$ and $\mathrm{Mn}$ were not examined; irreversible adsorption of Fe did not lead to selective step decoration, and it was assumed these adatoms would adsorb as their respective hydroxides (and not as metal adatoms), as on $\operatorname{Pt}(111)$ (ref. ${ }^{6}$ ).

Electrochemical measurements. Electrochemical measurements were performed with a VSP-300 potentiostat from Biologic. A typical electrochemical experiment involved measuring a 'blank' CV (between 0.06 and $0.8 \mathrm{~V}_{\mathrm{RHE}}$ ) in either $0.1 \mathrm{M} \mathrm{HClO}_{4}$ or $0.1 \mathrm{M} \mathrm{NaOH}$ to confirm, via the voltammetric response, the cleanliness of the electrolyte and the quality of the annealing procedure. Then the HER activity of the pristine electrode was measured in $0.1 \mathrm{M} \mathrm{NaOH}$ by linear sweep or cyclic voltammetry from -0.2 to $0.7 \mathrm{~V}_{\mathrm{RHE}}$. After this, the electrode was transferred to a perchloric acid electrolyte that contained the desired transition metal ion and cycled to deposit the metal atom at the step edge. The electrode, with the step edge decorated with the various metal atoms, was then transferred to the alkaline electrolyte and the HER activity measured (using the same potential limits as for the pristine electrode, except for $\mathrm{Mo}$ and $\mathrm{Re}$ on $\mathrm{Pt}(553)$ as they were found to desorb above $\sim 0.3 \mathrm{~V}_{\mathrm{RHE}}$, and so the upper potential limit was set to $0.2 \mathrm{~V}_{\mathrm{RHE}}$ ). For the Mo- and Re-decorated Pt(553), the transfer from the acidic electrolyte after deposition to the alkaline electrolyte to measure the HER activity was performed in a hydrogen atmosphere to retain the Mo or Re at the step edge. The coverage of the adsorbed adatoms was calculated by determining the fraction of the charge under the step peak that was blocked after adsorption. This charge was measured between 0.21 and $0.33 \mathrm{~V}_{\mathrm{RHE}}$ in the alkaline electrolyte ( $\mathrm{Re}, \mathrm{Ru}, \mathrm{Rh}$ and $\mathrm{Ag}$ ) and between 0.1 and $0.19 \mathrm{~V}_{\mathrm{RHE}}$ in the acid electrolyte (Mo). 
The kinetics of the hydrogen underpotential deposition were measured using electrochemical impedance spectroscopy. Impedance spectra were measured with frequencies from $10^{4}$ to $0.5 \mathrm{~Hz}$ with an amplitude of $5 \mathrm{mV}$. An equivalent circuit was fitted to the impedance spectra using the EIS Spectrum Analyser ${ }^{38}$. The equivalent circuit used was identical to that used by Schouten et al. to examine hydrogen and hydroxide adsorption on $\mathrm{Pt}(111)$ and stepped platinum ${ }^{39}$

Computational details. DFT calculations were performed using the Vienna Ab initio Simulations Package ${ }^{40-42}$. A plane wave basis set was used with a cutoff energy of $450 \mathrm{eV}$. The PW91 exchange-correlation functional was used ${ }^{43}$, and the ion core potentials were modelled following the projector augmented wave (PAW) approach ${ }^{44,45}$. Structural relaxations were performed until the forces on each of the atoms were below $0.02 \mathrm{eV} \AA^{-1}$. The Pt(111) surface was modelled with a $3 \times 3$ unit cell and the $\mathrm{Pt}(553)$ surface was modelled with a $3 \times 1$ unit cell, with three Pt step atoms. Monkhorst-Pack mesh $k$-space sampling grids were used $^{46}$, with $5 \times 5 \times 1$ and $7 \times 7 \times 1$ sampling grids used for $\mathrm{Pt}(111)$ and $\mathrm{Pt}(553)$, respectively. $\mathrm{Pt}(111)$ and $\mathrm{Pt}(553)$ were modelled as a four-layer and five-layer slab, respectively, with the bottom two layers frozen at the experimentally measured lattice constant, $3.92 \AA$ (ref. ${ }^{47}$ ). Dipole corrections were included in the surface normal direction ${ }^{48}$. Activation barriers for water dissociation were calculated using the climbing image-nudged elastic band method ${ }^{49}$. The TS was identified when the forces on all the atoms and the tangent force on the highest-energy image were below $0.02 \mathrm{eV}^{-1}$, and the tangent forces on the preceding images were opposite in sign to those on the images after the TS. All TS also showed one imaginary vibrational frequency along the reaction coordinate. The adsorption energy of hydrogen and hydroxide were also calculated using DFT for the adsorption onto a PtRu(111) alloy surface, as well as on and/or near a $\mathrm{Ru}^{*}$ cluster and/or island on Pt(111) (shown in Fig. 5). Relatively simple models were used to approximate the adsorption energetics. The $\mathrm{PtRu}(111)$ surface was modelled in a $3 \times 3$ unit cell with a five-layer slab, with the bottom two layers frozen at the DFT-optimized lattice constant of $3.9 \AA$ (compared with a DFT-optimized lattice constant for Pt of $3.985 \AA$ ). A $7 \times 7 \times 1 k$-point mesh was used. As there were an odd number of atoms in the slab, the ratio of $\mathrm{Pt}$ to $\mathrm{Ru}$ was $\mathrm{Pt}_{22} \mathrm{Ru}_{23}$. The adsorption of hydroxide was evaluated at a low coverage $(1 / 9 \mathrm{ML})$ in the presence of two co-adsorbed water molecules and a low coverage of co-adsorbed $\mathrm{Na}^{*}(1 / 9 \mathrm{ML})$. The adsorption of hydrogen was evaluated in the absence and the presence of co-adsorbed hydroxide, water and cation; the adsorption energy shown in Fig. 5 was calculated in the presence these co-adsorbates (as this was found to weaken hydrogen adsorption). Adsorption onto a $\mathrm{Ru}^{*}$ cluster was evaluated on a seven-atom $\mathrm{Ru}^{*}$ (single-layer) island adsorbed on a four-layer $4 \times 4 \mathrm{Pt}(111)$ slab (modelled using a $3 \times 3 \times 1$ $k$-point mesh). The adsorption of a single hydroxyl molecule was examined on the upper edge of the $\mathrm{Ru}^{*}$ cluster in the presence of five co-adsorbed water molecules and one co-adsorbed $\mathrm{Na}^{*}$. The adsorption of hydrogen was evaluated on the $\mathrm{Pt}(111)$ surface on a Pt site neighbouring the $\mathrm{Ru}^{*}$ island in the presence of co-adsorbed hydroxide, water, and cation.

Computational details - thermodynamics and kinetics. $\mathrm{OH}^{*}$ adsorption energy. The hydroxide adsorption energy was calculated following the reaction given in equation (10):

$$
3 \mathrm{H}_{2} \mathrm{O}^{*} \rightarrow \mathrm{OH}^{*} 2 \mathrm{H}_{2} \mathrm{O}^{*}+\mathrm{H}^{+}+e^{-}
$$

where $\mathrm{H}_{2} \mathrm{O}^{*}$ is water that is adsorbed on or close to the surface (or specifically the step on $\mathrm{Pt}(553)$ ) and $\mathrm{OH}^{\star} 2 \mathrm{H}_{2} \mathrm{O}^{*}$ is hydroxide adsorbed on the step (or on the $\mathrm{Pt}(111)$ surface) hydrogen bonded to two co-adsorbed water molecules. This stoichiometry in the $3 \times 3$ unit cell on $\mathrm{Pt}(111)$ gives an $\mathrm{OH}^{*}$ coverage of 1/9 ML and in the $3 \times 1$ unit cell of $\mathrm{Pt}(553)$ an $\mathrm{OH}^{*}$ coverage of $1 / 3 \mathrm{ML}$, defined as $\mathrm{OH}^{*}$ per Pt step atom. Adsorption was examined in the presence of a co-adsorbed and/ or near-surface sodium ion (at 1/9 ML on Pt(111) and 1/3 ML on Pt(553)), located near the step edge, as we have found previously that near-surface cations affect the adsorption strength of the hydroxide $e^{25}$. Our previous work also highlighted the importance of including co-adsorbed water molecule ${ }^{50}$. We chose near-surface (adsorbed) water as our reference state (instead of solution-phase water or hydroxide) as this minimizes the impact from errors in describing the metal-water interaction (because, in part, DFT poorly captures van der Waals interactions) in the calculated $\mathrm{OH}^{*}$ adsorption energy.

The free energy of adsorption of hydroxide was calculated using equation (11):

$$
\Delta G=G_{\mathrm{OH}^{*} 2 \mathrm{H}_{2} \mathrm{O}^{*}}+G_{\mathrm{H}^{+}}-|e| U-G_{3 \mathrm{H}_{2} \mathrm{O}^{*}}-|e|\left(U-U_{\mathrm{PZC}}\right) \frac{\Delta \mu}{d}
$$

where $G_{\mathrm{OH}^{*} 2 \mathrm{H}_{2} \mathrm{O}^{*}}$ is the free energy of co-adsorbed hydroxide and water, $G_{3 \mathrm{H}_{2}} \mathrm{O}^{*}$ is the free energy of adsorbed and/or near-surface water, $G_{\mathrm{H}^{+}}$is the free energy of an aqueous proton and $|e| U$ the free energy of an electron. The last term represents a correction to the free energy based on the interaction of the near-surface electric field with the surface normal dipole moment. $\Delta \mu$ is the change in surface normal dipole moment (product-reactant), $d$ the thickness of the Helmholtz layer (taken to be $3 \AA$ (ref. ${ }^{51}$ ) and $U_{\text {PZC }}$ the PZC of the surface (taken to be $0 \mathrm{~V}_{\mathrm{RHE}}$ ).
The free energies of the adsorbed species were calculated using equation (12):

$$
G_{X^{*}}=E_{X^{*}}^{\mathrm{DFT}}+\mathrm{ZPVE}-T S_{X^{*}}^{\mathrm{vib}}+E^{\mathrm{vib}}
$$

where $E_{X}^{\mathrm{DFT}}$ is the DFT-calculated internal energy at $0 \mathrm{~K}$, ZPVE the zero-point vibrational energy, $S_{X^{*}}^{\text {vib }}$ the vibrational entropy of the adsorbed species $X^{\star}$ (calculated assuming the phonon modes of the surface are unperturbed by adsorption) and $E^{\mathrm{vib}}$ the internal vibrational energy.

The free energies of the proton and electron are calculated following the computational hydrogen electrode method ${ }^{51}$, in which they are obtained from the free energy of hydrogen gas. The free energy of hydrogen gas is calculated following equation (13):

$$
G_{\mathrm{H}_{2}}=E_{\mathrm{H}_{2}}^{\mathrm{DFT}}+\mathrm{ZPVE}+E_{\mathrm{int}}-T S_{\mathrm{H}_{2}}+\mathrm{PV}
$$

where $S_{\mathrm{H}_{2}}$ includes vibrational, rotational and translational entropy for gas-phase hydrogen, $E_{\text {int }}$ includes all vibrational, rotational and translational internal energy for gas-phase hydrogen, and $\mathrm{PV}$ is calculated as $k_{\mathrm{B}} T$. All the calculations were performed at $1 \mathrm{~atm}, 300 \mathrm{~K}$ and $1 \mathrm{M}$ (unless otherwise noted).

All the hydroxide adsorption energies are reported at an applied potential of $0 \mathrm{~V}_{\mathrm{RHE}}$ unless otherwise noted.

$H^{*}$ adsorption energy. The free energy for the adsorption of hydrogen was calculated in a similar manner as that for hydroxide, using the reaction in equation (14):

$$
\mathrm{H}^{+}+e^{-}+{ }^{*} \rightarrow \mathrm{H}^{*}
$$

To calculate the adsorption energy of hydrogen as a function of coverage on the terrace of $\mathrm{Pt}(553)$, multiple adsorbates were included in the $3 \times 1$ unit cell.

Potential independent activation energy. Potential independent activation energies for the chemical dissociation of water were calculated using the climbing image nudged elastic band method, in which near-surface and/or adsorbed water was dissociated to adsorbed hydrogen and adsorbed hydroxide, in the presence of near-surface solvation (additional explicit water molecules) and near-surface alkali metal cation $\left(\mathrm{Na}^{\star}\right)$. On the bare and decorated $\mathrm{Pt}(553)$, all the activation energies were calculated for the formation of adsorbed hydroxide on the step and adsorbed hydrogen on the terrace (close to the bottom of the step edge, the weakest binding), as this gave the lowest barrier for dissociation on the bare surface (as the step sites give the strongest binding for $\mathrm{OH}^{*}$ and the terrace sites close to the bottom of the step give the weakest binding for $\mathrm{H}^{*}$ ).

Potential dependent activation energy. Potential dependent activation energies for water dissociation into solution-phase hydroxide (electrochemical) were calculated by taking the barrier calculated by the TS search for water dissociation to adsorbed hydroxide (chemical) as the barrier for the electrochemical dissociation at the potential where the FS (adsorbed hydroxide, $\mathrm{OH}^{*}+2 \mathrm{H}_{2} \mathrm{O}^{*}+\mathrm{Na}^{*}+\mathrm{H}^{*}$ ) was in equilibrium with solution-phase hydroxide ${ }^{28}$. The potential at which the FS is in equilibrium with the solution-phase hydroxide was calculated following reaction (15) (with reference to adsorbed water, instead of to solution-phase hydroxide explicitly, as done for the $\mathrm{OH}^{*}$ adsorption energy in equation (11)), using equation (11) but solving for the potential that gives a free energy change of adsorption of 0 . The activation energy at $0 \mathrm{~V}_{\mathrm{RHE}}$ (the equilibrium potential for hydrogen evolution) was then calculated by extrapolating the activation barrier at the equilibrium potential (between adsorbed and solution-phase hydroxide) to $0 \mathrm{~V}_{\mathrm{RHE}}$ using the Butler-Volmer law ${ }^{28}$. This is shown in equation (16):

$$
\begin{gathered}
3 \mathrm{H}_{2} \mathrm{O}^{*} \mathrm{H}^{*} \mathrm{Na}^{*} \rightarrow \mathrm{OH}^{*} 2 \mathrm{H}_{2} \mathrm{O}^{*} \mathrm{H}^{*} \mathrm{Na}^{*}+\mathrm{H}^{+}+e^{-} \\
\Delta E_{\text {electrochemical }}^{\mathrm{TS}}(V)=\Delta E_{\text {chemical }}^{\mathrm{TS}}+\alpha e_{0}\left(V-U_{\mathrm{OH}^{*} \text { ads }}^{0}\right)
\end{gathered}
$$

where $\Delta E_{\text {electrochemical }}^{\mathrm{TS}}(V)$ is the activation energy for the electrochemical dissociation of water to solution-phase hydroxide at the desired potential, $V$, $\Delta E_{\text {chemical }}^{\mathrm{TS}}$ is the activation energy for the chemical dissociation of water to adsorbed hydrogen and adsorbed hydroxide for $V=U_{\mathrm{OH}^{*} \text { ads }}^{0}, U_{\mathrm{OH}^{*} \text { ads }}^{0}$ is the equilibrium potential (defined as the potential that gives a free energy change of reaction of 0 ) for hydroxide adsorption in the presence of adsorbed hydrogen (reaction (15)) and $\alpha$ is the transfer coefficient (which can vary from 0 to 1 ), assumed here to be 0.5 .

Simulated rate of hydrogen evolution. In an alkaline electrolyte, hydrogen evolution comprises elementary steps that dissociate water to produce adsorbed hydrogen (Volmer), recombine adsorbed hydrogen to form hydrogen gas (Tafel) and/or an elementary step that involves both (Heyrovsky). Our DFT calculations show that the barrier to dissociate water (modelled as a Volmer step) correlates with how strongly the electrode surface binds hydroxide (presumably a Heyrovsky-type mechanism might show the same dependence), regardless of whether hydroxide is adsorbed in the product state or not. The experimentally measured correlation between hydroxide binding strength and HER activity on the too-weak binding 
side of the volcano (where stronger $\mathrm{OH}^{*}$ binding leads to higher HER activity), can then be readily explained by taking water dissociation to give the solution-phase hydroxide as the RDS. We consider only a Volmer step and not a Heyrovsky step for simplicity. The overall mechanism for the weak-binding side of the volcano is shown in equations (17) and (18):

$$
\begin{gathered}
\mathrm{H}_{2} \mathrm{O}+{ }^{*}+e^{-} \rightarrow \mathrm{H}^{*}+\mathrm{OH}^{-} \text {RD } \\
2 \mathrm{H}^{*} \rightarrow \mathrm{H}_{2}+2^{*} \text { Fast }
\end{gathered}
$$

Taking equation (17) as the RDS and equation (18) to proceed rapidly allows us to calculate the rate on the too-weak binding side of the volcano, as shown in equations (19) and (20):

$$
\begin{gathered}
\text { Rate }=k_{\mathrm{W}}\left(1-\theta_{\mathrm{H}^{*}}\right) \\
\text { Rate }=A \exp \left(\frac{-\Delta G_{\mathrm{W}}^{\mathrm{TS}}}{R T}\right) \frac{1}{1+\exp \left(\frac{-\Delta G_{\mathrm{H}^{*}}}{R T}\right)}
\end{gathered}
$$

where $k_{\mathrm{W}}$ is the rate constant for water dissociation, $\theta_{\mathrm{H}^{*}}$ the coverage of adsorbed hydrogen, $A$ the pre-exponential factor and $\Delta G_{\mathrm{W}}^{\mathrm{TS}}$ the barrier for water dissociation (which we approximate using the $0 \mathrm{~K}$ enthalpic barrier). We calculated both the (enthalpic) barrier for water dissociation and the adsorption free energy of hydrogen using DFT, and assumed a pre-exponential factor. As a simplification, we assumed the pre-exponential factor is the same for both sides of the volcano, and fit this as a free parameter to the experiment (to reproduce the rate of HER on $\mathrm{Ag}^{\star}$ on $\mathrm{Pt}(553)$, which gave a pre-exponential factor of $\left.3 \times 10^{10} \mathrm{~s}^{-1}\right)$. We note that, as the rate is simulated at $0 \mathrm{~V}_{\mathrm{RHE}}$ and experimentally measured at $-0.038 \mathrm{~V}_{\mathrm{RHE}}$, this pre-exponential factor is not equivalent to what one might calculate from experiment (or DFT) alone. To calculate the water dissociation barrier at any hydroxide adsorption free energy (as we plot the rate as a function of hydroxide adsorption energy), we fitted a line through the data shown in Fig. 3a, where we plot the DFT-calculated electrochemical water dissociation barrier (to solution-phase hydroxide) as a function of hydroxide adsorption strength on our decorated $\mathrm{Pt}(553)$ surface (to give a slope of 0.27 and intercept of $0.31 \mathrm{eV}$ ). We note that the near-surface solvation structure for $\mathrm{OH}^{*}$ in the product state of the dissociation reaction for $\mathrm{Ag}^{*}$ on $\mathrm{Pt}(553)$ is not the same as that used to calculate the adsorption free energy of hydroxide on $\mathrm{Ag}^{*}$ shown in the volcano plots in Figs. 2 and 4 (and therefore the $\mathrm{OH}^{*}$ adsorption strength shown in Fig. 3 a for $\mathrm{Ag}^{*}$ on $\mathrm{Pt}(553)$ is much higher (less favourable)). Removing $\mathrm{Ag}^{\star}$ on $\mathrm{Pt}(553)$ from Fig. $3 \mathrm{a}$ does not substantially affect the calculated slope and intercept.

In Fig. 4, we show the rate calculated at an applied overpotential of $0 \mathrm{~V}$ (which effectively gives the exchange current density for hydrogen evolution). We also calculated the rate based on a surface area (to convert from inverse seconds to units of $\mathrm{mA} \mathrm{cm}^{-2}$ ) of $1.5 \times 10^{15}$ atoms cm${ }^{-2}$ (which yields a charge of $240 \mu \mathrm{C} \mathrm{cm}^{-2}$ for one electron transferred per surface atom) for both $\mathrm{Pt}(111)$ and $\mathrm{Pt}(553)$, as a simplification. To compare with the experimentally measured activity on $\mathrm{Pt}(553)$, as we found the steps to be more active than the terrace sites, we multiplied our experimentally measured activities by a factor of five (the ratio of terrace sites to step sites) in Fig. 4 in which we compare the DFT-simulated rate with the experimentally measured rate. For the too-strong binding side of the volcano, the RDS must be the desorption of adsorbed hydroxide (which would become slower with increasing hydroxide binding strength). The mechanism is then as in equations (21)-(23):

$$
\mathrm{H}_{2} \mathrm{O}+2^{*} \rightleftarrows \mathrm{H}^{*}+\mathrm{OH}^{*} \text { In equilibrium }
$$

$$
\mathrm{OH}^{*}+e^{-} \rightarrow \mathrm{OH}^{-} \text {RDS }
$$

$$
2 \mathrm{H}^{*} \rightarrow \mathrm{H}_{2}+2^{*} \text { Fast }
$$

Assuming that the coverage of the adsorbed hydrogen is low because the hydrogen evolution is fast, and assuming that the barrier for hydroxide desorption is equal to the free energy of desorption, we can calculate the maximum rate for hydrogen evolution on the too-strong binding side of the volcano using equation (24):

$$
\text { Rate }=A \exp \left(\frac{\Delta G_{\mathrm{OH}^{*}}}{R T}\right)
$$

Taking the barrier as equal to the free energy of hydroxide desorption yields an upper-bound on the rate of hydroxide desorption, any barrier beyond the reaction energy would further decrease the rate. It is interesting to note that the rate of hydroxide adsorption/desorption on $\mathrm{Pt}(111)$ in both acid and alkaline electrolytes is so fast that the kinetics cannot (or at least, have not, as of yet) be accurately measured with impedance spectroscopy (suggesting rates faster than that of hydrogen adsorption/desorption in an acid electrolyte on $\operatorname{Pt}(111))^{39,52}$. This suggests that on $\mathrm{Pt}(111)$, at equilibrium, where the reaction energy for hydroxide adsorption/desorption $\left(\Delta G_{\mathrm{OH}^{*}}\right)$ is zero, the barrier is small (and yields a high rate of adsorption/desorption).

Simulated 3D hydrogen and hydroxide volcano plot. To simulate the rate of hydrogen evolution as a function of both hydrogen and hydroxide binding strength, we first need the rate of water dissociation, or the activation barrier for water dissociation, as a function of both the hydrogen and hydroxide binding strength. In our DFT calculations, we only examined the effect of hydroxide binding strength on the water dissociation barrier. Tsai et al. used DFT to calculate the activation barrier for water dissociation across a variety of transition metal (111), (100) and (211) surfaces, and showed that the barriers are a linear function of the hydrogen and hydroxide binding strength ${ }^{34}$. (For the (211) surfaces, this linear fit follows $\Delta E_{\mathrm{TS}}=0.72 \Delta E_{\mathrm{H}}+0.73 \Delta E_{\mathrm{OH}}+1.08 \mathrm{eV}\left(\right.$ ref. $\left.\left.{ }^{34}\right)\right)$. Using this linear correlation, we can calculate the activation energy for water dissociation for any hydrogen and hydroxide binding strength. However, their work (as it was studied for thermal water dissociation) does not include the effects of near-surface solvation, near-surface alkali metal cations or electrochemical potential on the activation energy.

To convert their reported chemical activation barriers for water dissociation into an electrochemical, potential-dependent barrier, we used the same method as described above and added $\alpha e_{0}\left(V-U_{\mathrm{OH}^{*}}^{0}\right)$ (assuming an $\alpha$ of $0.5)$ to the chemical barrier to extrapolate this barrier to the electrochemical barrier at a potential $(V)$ of $0 \mathrm{~V}_{\mathrm{RHE}}$ (taking the hydroxide adsorption energy as an approximation to the free energy, and therefore the electrochemical adsorption potential). We did this using their reported data on the stepped (211) surfaces, and we then fitted a linear correlation between the electrochemical activation energy and the hydrogen and hydroxide binding strengths, with the slope for the dependence on hydrogen adsorption strength kept constant (the same as for their reported linear fit to the chemical barrier) (to yield $\left.\Delta E_{\mathrm{TS}}=0.72 \Delta E_{\mathrm{H}}+0.51 \Delta E_{\mathrm{OH}}+1.91\right)$. To approximately account for the effects of solvation and alkali metal cation, we shifted the intercept of this linear fit to best reproduce the activation energies we calculated with DFT for $\mathrm{Pt}(553)$ and $\mathrm{Pt}(111)$ at the hydrogen and hydroxide adsorption free energies we calculated (to finally give $\Delta G_{\mathrm{TS}}=0.72 \Delta G_{\mathrm{H}}+0.51 \Delta G_{\mathrm{OH}}+0.38$ ).

The other possible RDS, aside from water dissociation, are hydroxide desorption (for too-strong hydroxide binding) and hydrogen desorption and/or recombination (as $\mathrm{H}_{2}$ gas, for too-strong hydrogen binding). We assumed the activation energy for each of these steps is equal to the desorption energy of hydroxide and hydrogen, respectively. This should represent the minimum possible activation energy, and therefore the highest possible rate. To calculate the rate, we assumed a constant pre-exponential factor, which is identical for each RDS.

\section{Data availability}

All the data are available in the main text and Supplementary Information. Additional datasets related to this study are available from the corresponding author upon reasonable request. Source data are provided with this paper.

Received: 18 April 2020; Accepted: 16 September 2020; Published online: 19 October 2020

\section{References}

1. Trasatti, S. Work function, electronegativity, and electrochemical behaviour of metals: III. Electrolytic hydrogen evolution in acid solutions. J. Electroanal. Chem. Interfacial Electrochem. 39, 163-184 (1972).

2. Nørskov, J. K. et al. Trends in the exchange current for hydrogen evolution. J. Electrochem. Soc. 152, J23-J26 (2005).

3. Strmcnik, D. et al. Improving the hydrogen oxidation reaction rate by promotion of hydroxyl adsorption. Nat. Chem. 5, 300-306 (2013).

4. Wang, Y. et al. Pt-Ru catalyzed hydrogen oxidation in alkaline media: oxophilic effect or electronic effect? Energy Environ. Sci. 8, 177-181 (2015).

5. Schwämmlein, J. N. et al. Origin of superior HOR/HER activity of bimetallic $\mathrm{Pt}-\mathrm{Ru}$ catalysts in alkaline media identified via Ru@Pt core-shell Nanoparticles. J. Electrochem. Soc. 165, H229-H239 (2018).

6. Subbaraman, R. et al. Trends in activity for the water electrolyser reactions on $3 d \mathrm{M}(\mathrm{Ni}, \mathrm{Co}, \mathrm{Fe}, \mathrm{Mn})$ hydr(oxy)oxide catalysts. Nat. Mater. 11, 550-557 (2012).

7. Zeng, Z., Chang, K.-C., Kubal, J., Markovic, N. M. \& Greeley, J. Stabilization of ultrathin (hydroxy)oxide films on transition metal substrates for electrochemical energy conversion. Nat. Energy 2, 17070 (2017).

8. Sheng, W., Gasteiger, H. A. \& Shao-Horn, Y. Hydrogen oxidation and evolution reaction kinetics on platinum: acid vs alkaline electrolytes. J. Electrochem. Soc. 157, B1529-B1536 (2010).

9. Durst, J. et al. New insights into the electrochemical hydrogen oxidation and evolution reaction mechanism. Energy Environ. Sci. 7, 2255-2260 (2014).

10. Zheng, J., Sheng, W., Zhuang, Z., Xu, B. \& Yan, Y. Universal dependence of hydrogen oxidation and evolution reaction activity of platinum-group metals on $\mathrm{pH}$ and hydrogen binding energy. Sci. Adv. 2, e1501602 (2016).

11. Ledezma-Yanez, I. et al. Interfacial water reorganization as a $\mathrm{pH}$-dependent descriptor of the hydrogen evolution rate on platinum electrodes. Nat. Energy 2, 17031 (2017) 
12. Zheng, Y., Jiao, Y., Vasileff, A. \& Qiao, S.-Z. The hydrogen evolution reaction in alkaline solution: from theory, single crystal models, to practical electrocatalysts. Angew. Chem. Int. Ed. 57, 7568-7579 (2018).

13. Dubouis, N. \& Grimaud, A. The hydrogen evolution reaction: from material to interfacial descriptors. Chem. Sci. 10, 9165-9181 (2019).

14. Ryu, J. \& Surendranath, Y. Tracking electrical fields at the $\mathrm{Pt} / \mathrm{H}_{2} \mathrm{O}$ interface during hydrogen catalysis. J. Am. Chem. Soc. 141, 15524-15531 (2019).

15. Sarabia, F. J., Sebastián-Pascual, P., Koper, M. T. M., Climent, V. \& Feliu, J. M. Effect of the interfacial water structure on the hydrogen evolution reaction on $\mathrm{Pt}(111)$ modified with different nickel hydroxide coverages in alkaline media. ACS Appl. Mater. Interfaces 11, 613-623 (2019).

16. Zeradjanin, A. R. et al. Balanced work function as a driver for facile hydrogen evolution reaction-comprehension and experimental assessment of interfacial catalytic descriptor. Phys. Chem. Chem. Phys. 19, 17019-17027 (2017).

17. Li, J. et al. Experimental proof of the bifunctional mechanism for the hydrogen oxidation in alkaline media. Angew. Chem. Int. Ed. 56, 15594-15598 (2017)

18. Liu, E. et al. Unifying the hydrogen evolution and oxidation reactions kinetics in base by identifying the catalytic roles of hydroxyl-water-cation adducts. J. Am. Chem. Soc. 141, 3232-3239 (2019).

19. Cong, Y. et al. Uniform $\mathrm{Pd}_{0.33} \mathrm{Ir}_{0.67}$ nanoparticles supported on nitrogen-doped carbon with remarkable activity toward the alkaline hydrogen oxidation reaction. J. Mater. Chem. A 7, 3161-3169 (2019).

20. Qiu, Y. et al. BCC-phased PdCu alloy as a highly active electrocatalyst for hydrogen oxidation in alkaline electrolytes. J. Am. Chem. Soc. 140, 16580-16588 (2018)

21. Intikhab, S., Snyder, J. D. \& Tang, M. H. Adsorbed hydroxide does not participate in the Volmer step of alkaline hydrogen electrocatalysis. ACS Catal. 7, 8314-8319 (2017).

22. Rebollar, L., Intikhab, S., Snyder, J. D. \& Tang, M. H. Determining the viability of hydroxide-mediated bifunctional HER/HOR mechanisms through single-crystal voltammetry and microkinetic modeling. J. Electrochem. Soc. 165, J3209-J3221 (2018).

23. Massong, H., Wang, H., Samjeské, G. \& Baltruschat, H. The co-catalytic effect of $\mathrm{Sn}, \mathrm{Ru}$ and Mo decorating steps of $\mathrm{Pt}(111)$ vicinal electrode surfaces on the oxidation of CO. Electrochim. Acta 46, 701-707 (2001).

24. Domke, K. F., Xiao, X.-Y. \& Baltruschat, H. The formation of two Ag UPD layers on stepped Pt single crystal electrodes and their restructuring by co-adsorption of CO. Electrochim. Acta 54, 4829-4836 (2009).

25. Chen, X., McCrum, I. T., Schwarz, K. A., Janik, M. J. \& Koper, M. T. M. Co-adsorption of cations as the cause of the apparent $\mathrm{pH}$ dependence of hydrogen adsorption on a stepped platinum single-crystal electrode. Angew. Chem. Int. Ed. 56, 15025-15029 (2017)

26. Farias, M. J. S., Cheuquepan, W., Camara, G. A. \& Feliu, J. M. Disentangling catalytic activity at terrace and step sites on selectively Ru-modified well-ordered Pt surfaces probed by $\mathrm{CO}$ electro-oxidation. ACS Catal. 6 2997-3007 (2016).

27. Markovića, N. M., Sarraf, S. T., Gasteiger, H. A. \& Ross, P. N. Hydrogen electrochemistry on platinum low-index single-crystal surfaces in alkaline solution. J. Chem. Soc. Faraday Trans. 92, 3719-3725 (1996).

28. Akhade, S. A., Bernstein, N. J., Esopi, M. R., Regula, M. J. \& Janik, M. J. A simple method to approximate electrode potential-dependent activation energies using density functional theory. Catal. Today 288, 63-73 (2017).

29. Lamoureux, P. S., Singh, A. R. \& Chan, K. pH effects on hydrogen evolution and oxidation over $\mathrm{Pt}(111)$ : insights from first-principles. ACS Catal. 9 6194-6201 (2019).

30. Eigen, M. \& de Maeyer, L. Self-dissociation and protonic charge transport in water and ice. Proc. R. Soc. Lond. A 247, 505-533 (1958)

31. Durst, J., Simon, C., Hasché, F. \& Gasteiger, H. A. Hydrogen oxidation and evolution reaction kinetics on carbon supported $\mathrm{Pt}, \mathrm{Ir}, \mathrm{Rh}$, and $\mathrm{Pd}$ electrocatalysts in acidic media. J. Electrochem. Soc. 162, F190-F203 (2015).

32. Wang, L. et al. Platinum-nickel hydroxide nanocomposites for electrocatalytic reduction of water. Nano Energy 31, 456-461 (2017).

33. Koper, M. T. M. A basic solution. Nat. Chem. 5, 255-256 (2013).

34. Tsai, C. et al. Direct water decomposition on transition metal surfaces: structural dependence and catalytic screening. Catal. Lett. 146, 718-724 (2016).

35. Intikhab, S. et al. Exploiting dynamic water structure and structural sensitivity for nanoscale electrocatalyst design. Nano Energy 64, 103963 (2019).

36. Strmcnik, D., Lopes, P. P., Genorio, B., Stamenkovic, V. R. \& Markovic, N. M. Design principles for hydrogen evolution reaction catalyst materials. Nano Energy 29, 29-36 (2016).
37. Clavilier, J., Faure, R., Guinet, G. \& Durand, R. Preparation of monocrystalline Pt microelectrodes and electrochemical study of the plane surfaces cut in the direction of the $\{111\}$ and $\{110\}$ planes. J. Electroanal. Chem. Interfacial Electrochem. 107, 205-209 (1980).

38. Bondarenko, A. S. \& Ragoisha, G. A. Progress in Chemometrics Research 89-102 (Nova Science, 2005).

39. Schouten, K. J. P., van der Niet, M. J. T. C. \& Koper, M. T. M. Impedance spectroscopy of $\mathrm{H}$ and $\mathrm{OH}$ adsorption on stepped single-crystal platinum electrodes in alkaline and acidic media. Phys. Chem. Chem. Phys. 12, 15217-15224 (2010)

40. Kresse, G. \& Furthmüller, J. Efficient iterative schemes for ab initio total-energy calculations using a plane-wave basis set. Phys. Rev. B 54, 11169-11186 (1996)

41. Kresse, G. \& Furthmüller, J. Efficiency of ab-initio total energy calculations for metals and semiconductors using a plane-wave basis set. Comput. Mater. Sci. 6, 15-50 (1996)

42. Kresse, G. \& Hafner, J. Ab initio molecular dynamics for liquid metals. Phys. Rev. B 47, 558-561 (1993).

43. Perdew, J. P. et al. Atoms, molecules, solids, and surfaces: applications of the generalized gradient approximation for exchange and correlation. Phys. Rev. B 46, 6671-6687 (1992).

44. Blöchl, P. E. Projector augmented-wave method. Phys. Rev. B 50, 17953-17979 (1994)

45. Kresse, G. \& Joubert, D. From ultrasoft pseudopotentials to the projector augmented-wave method. Phys. Rev. B 59, 1758-1775 (1999).

46. Monkhorst, H. J. \& Pack, J. D. Special points for Brillouin-zone integrations. Phys. Rev. B 13, 5188-5192 (1976)

47. Kittel, C. Introduction to Solid State Physics 7th edn (Wiley, 2008).

48. Bengtsson, L. Dipole correction for surface supercell calculations. Phys. Rev. B 59, 12301-12304 (1999).

49. Henkelman, G., Uberuaga, B. P. \& Jónsson, H. A climbing image nudged elastic band method for finding saddle points and minimum energy paths. J. Chem. Phys. 113, 9901-9904 (2000).

50. McCrum, I. T. \& Janik, M. J. First principles simulations of cyclic voltammograms on stepped $\mathrm{Pt}(553)$ and $\mathrm{Pt}(533)$ electrode surfaces. ChemElectroChem 3, 1609-1617 (2016).

51. Nørskov, J. K. et al. Origin of the overpotential for oxygen reduction at a fuel-cell cathode. J. Phys. Chem. B 108, 17886-17892 (2004).

52. Sibert, E., Faure, R. \& Durand, R. High frequency impedance measurements on $\mathrm{Pt}(111)$ in sulphuric and perchloric acids. J. Electroanal. Chem. 515, 71-81 (2001).

53. Schmidt, T. J., Ross, P. N. \& Markovic, N. M. Temperature dependent surface electrochemistry on Pt single crystals in alkaline electrolytes: Part 2. The hydrogen evolution/oxidation reaction. J. Electroanal. Chem. 524-525, 252-260 (2002).

\section{Acknowledgements}

This project received funding from the European Union's Horizon 2020 research and innovation programme under the Marie Skłodowska-Curie grant agreement no. 707404. The use of supercomputing facilities at SURFsara was sponsored by NWO Physical Sciences, with financial support by NWO.

\section{Author contributions}

I.T.M. and M.T.M.K. designed the experimental plan. I.T.M. carried out the experiments, DFT simulations and data analysis. I.T.M. and M.T.M.K. wrote the manuscript.

\section{Competing interests}

The authors declare no competing interests.

\section{Additional information}

Supplementary information is available for this paper at https://doi.org/10.1038/ s41560-020-00710-8.

Correspondence and requests for materials should be addressed to M.T.M.K.

Reprints and permissions information is available at www.nature.com/reprints.

Publisher's note Springer Nature remains neutral with regard to jurisdictional claims in published maps and institutional affiliations.

(c) The Author(s), under exclusive licence to Springer Nature Limited 2020 